\title{
Ultra-Dense, Transparent and Resilient Ring-Tree Access Network using Coupler-Based Remote Nodes and Homodyne Transceivers
}

\author{
Josep M. Fàbrega, Student Member, IEEE, Josep Prat, Member, IEEE \\ Universitat Politècnica de Catalunya, Jordi Girona, 1-3; E-08034 Barcelona, Spain \\ Tel: +34934017179,Fax: +34934017200, e-mail: jmfabrega@tsc.upc.edu
}

\begin{abstract}
We propose an Ultra-Dense Wavelength Division Multiplex Passive Optical Network (UD-WDM-PON) based on homodyne detection in a totally transparent ring-tree implementation. It is based on time switching phase diversity homodyne detection and local oscillator reuse for upstream transmission. $4 \mathrm{GHz}$ spaced $1 \mathrm{~Gb} / \mathrm{s}$ streams were correctly received in a $30 \mathrm{~km}$ experimental deployment serving 1024 users.
\end{abstract}

Keywords: optical communication, access networks, coherent detection, optical communication terminals.

\section{INTRODUCTION}

Transparency is a key feature in optical fiber access networks, as the infrastructure is very costly to deploy. Typically, returns on investment in optical fiber outside plants are calculated for periods of 20 years, which means that the infrastructure should not require major modifications during this period of time. Additionally, easy upgradeability is a desirable feature as the network should be scalable to fulfill future requirements in terms of users and transmission capabilities.

At the same time, advanced multimedia applications increase transmission requirements, thus alternatives to classical Time Division Multiplex (TDM) PONs are appearing in order to increase available bandwidth [1]. WDM provides virtual point-to-point connections, so multiplies the effective bandwidth that the fiber can offer [2]. A significant step forward is UD-WDM, where wavelengths are separated by just a few $\mathrm{GHz}$ $(<10 \mathrm{GHz})$, increasing the number of channels that can be accommodated on a single fiber.

This paper presents an advanced network topology combining a central ring and secondary trees to transmit UD-WDM signals transparently. For the first time, it uses homodyne detection, to achieve very small channel spacing, excellent wavelength selectivity, and excellent sensitivity [3]-[4]. The network topology also offers resilience and easy upgradeability, as it is totally wavelength transparent. Consequently, the addition of new users is straightforward.

\section{NETWORK TOPOLOGY AND TRANSMISSION STRATEGIES}

As shown in Fig. 1, the network topology fiber, is based on a two-stage outside plant, with a primary couplerbased feeder ring and a secondary power-splitter-based distribution stage. The central ring multicasts all the wavelengths transmitted on the trunk fiber to the different networks sub-segments. Subsequently, the secondary power-splitters multicast the signals to the end users. At the customer premises equipment (CPE) side, the receiver selects the wavelength that has been assigned to establish a virtual point-to-point link. Even it is not explicitly shown in Fig. 1, this is a double fiber topology, with separate fibers for upstream and downstream.

This topology has some interesting features: total transparency and scalability. The addition of a new user simply requires the installation of an optical 1:2 coupler and the interface at Central Office (CO). Also, resilience is achieved naturally with the central ring, the bidirectional design of the $\mathrm{RN}$ and by means of an optical switch that connects the interfaces at the head-end with the branch of the ring that offers the best connectivity. This is shown in Fig. 1. In case of a fiber break, there is always a light path to reach all the remote nodes.

Transmission losses are increased due to the use of power couplers instead of wavelength multiplexers. This is the price to pay for total transparency and scalability. Homodyne detection can be used to overcome this problem, as it is known to offer much better sensitivity than direct detection systems.

A stack of thermally controlled lasers located at the CO generate all the wavelengths, which are Differential Phase Shift Keying (DPSK) modulated with downstream data and transmitted to the ONUs. Then, at the CPE, the tunable local oscillator selects the ONU's assigned channel and decodes downstream data by means of optical homodyning, IQ phase switching, differential demodulation and post-processing, following the steps described in [4]. This same optical oscillator carrier is then used for upstream transmission. It is modulated using again DPSK modulation format. At the $\mathrm{CO}$ a symmetric transceiver is implemented (see Fig. 2).

The main feature of the transceiver design used is its potential low cost. According to [5], the optical circuit used for reception (and transmission) can be integrated into an InP substrate. This may enable a potential low cost implementation of such solution. 


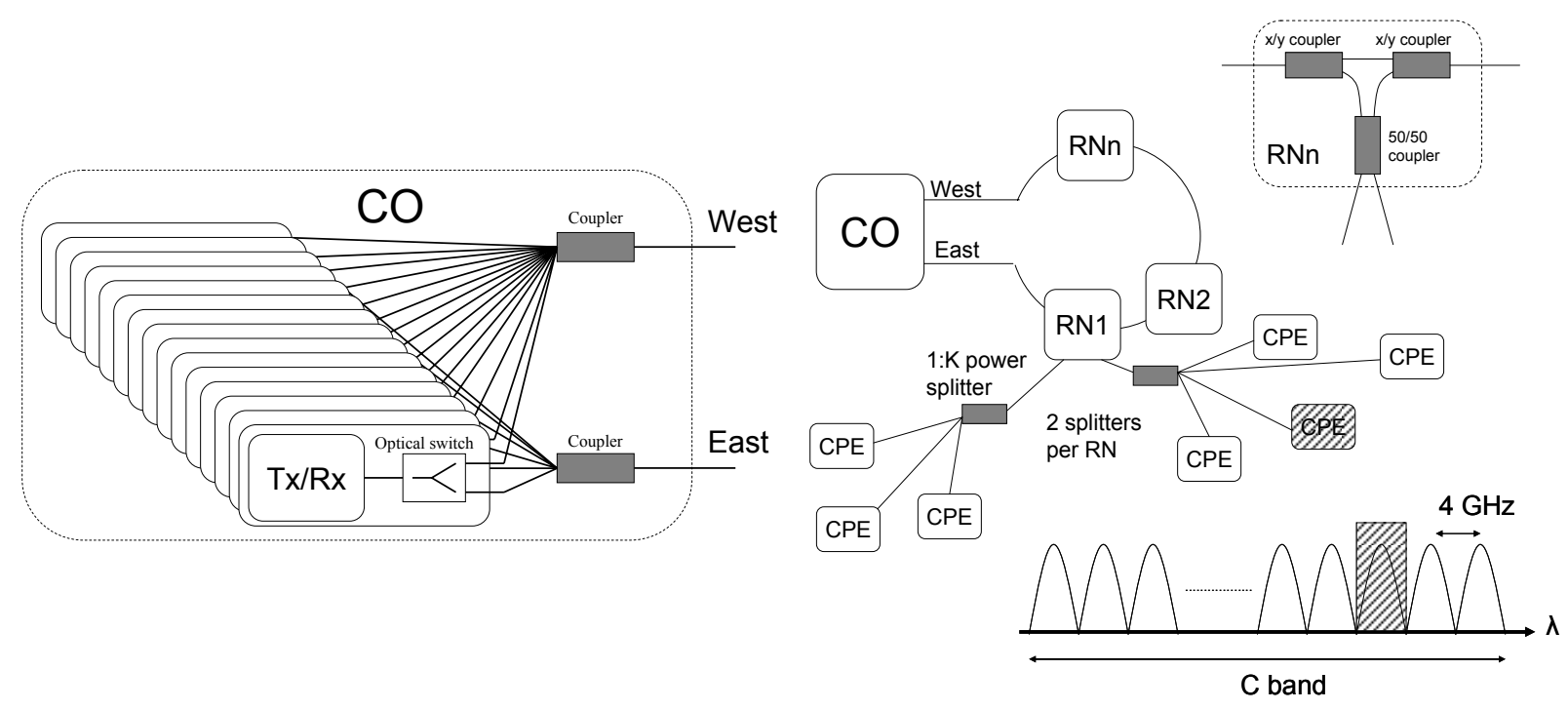

Figure 1. Central office scheme (left). Network topology and wavelength plan (right).

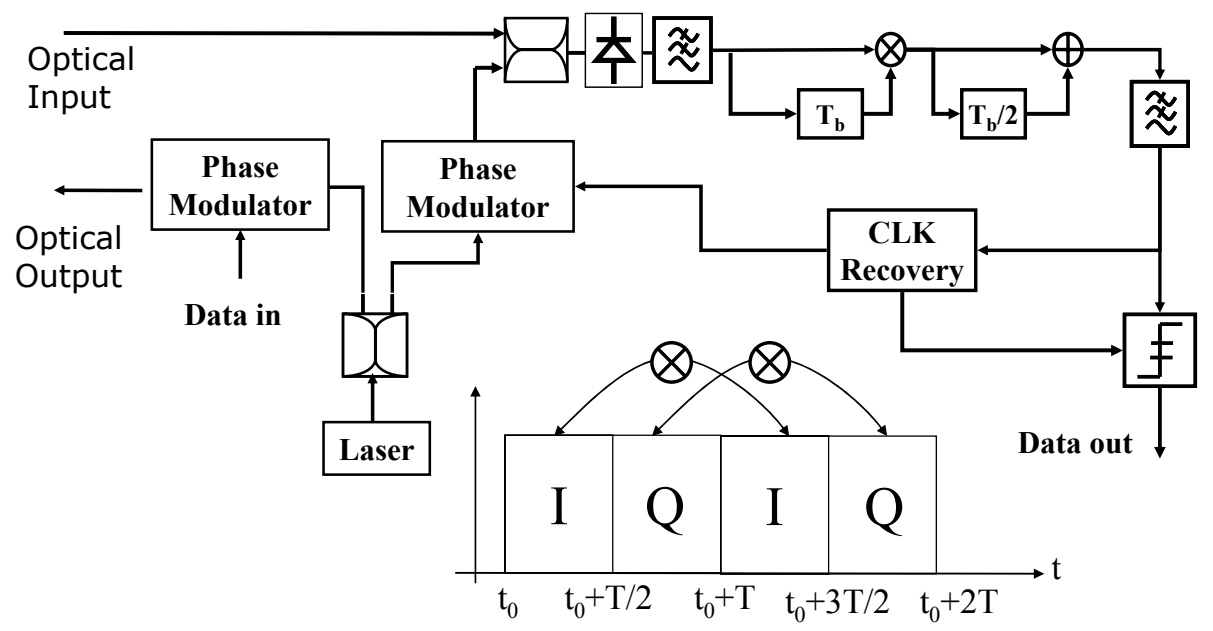

Figure 2. Transmission/reception subsystem.

The network that we have implemented is designed to offer connectivity to 1024 users (U) per fiber in a 4-node $(N)$ configuration with 8 secondary trees with 1:128 $(K)$ splitting factor. Other topologies are also possible taking into account that the total number of users is $U=2 \mathrm{NK}$ and that total link losses are:

$$
L=L_{\text {fiber }}+(N-1) \cdot 10 \cdot \log (x)+N \cdot L_{e x}+10 \cdot \log (y)+3+3 \cdot \log _{2}(K)
$$

where $x$ and $y$ are the remote node coupling factors (typically $x=0.9 ; y=0.1$ ), $L_{f i b e r}$ is the attenuation along the fiber and $L_{e x}$ are excess losses due to alignment and manufacturing imperfections of the components per remote node. A priori, the number of users is mainly limited by the available power budget.

Regarding available bandwidth in the proposed network, a 4-GHz channel spacing can be used, at $1 \mathrm{~Gb} / \mathrm{s}$ data rate, as demonstrated in [6]. Thus, 1024 channels can be easily accommodated in the overall C-band. So the network can potentially serve those 1024 users at the same time, offering a huge total capacity, of slightly more than $1 \mathrm{~Tb} / \mathrm{s}$.

A possible limit of the network is the total optical power that can be launched into the ring fiber. According IEC safety rules [7], that power should not exceed $1270 \mathrm{~mW}(\sim 31 \mathrm{dBm})$ at $1550 \mathrm{~nm}$. In the network plan described in Fig. 1, all the operative wavelengths are launched into the ring fiber at the same time. In the worst case, working in resilient mode, all 1024 wavelengths would be launched on the same side of the ring. Thus, the maximum power of each wavelength should not exceed $0.9 \mathrm{dBm}$.

\section{TRANSMISSION EXPERIMENTS AND DISCUSSION}

To demonstrate the feasibility of the network, proof-of-concept network experiments were carried out using the network setup detailed in Fig. 3 below. 
At the CO, a $1550 \mathrm{~nm}$ Distributed Feed-Back (DFB) commercial laser was externally DPSK modulated at $1 \mathrm{~Gb} / \mathrm{s}$, with a $\mathrm{LiNbO}_{3}$ phase modulator. The output power was of $0 \mathrm{dBm}$. To simulate the case in a UD-WDM scenario, a 4-GHz-spaced signal was also inserted, using another continuous wave laser. A $30 \mathrm{~km}$ fiber spool was used as the access trunk fiber to reach the first remote node $(L=5.2 \mathrm{~dB})$. This remote node $(\mathrm{RN})$ was based on two 90/10 couplers and a 3-dB coupler to connect to two access trees. Insertion losses of this device were measured to be $1.6 \mathrm{~dB}$ for pass-through signals and $13.2 \mathrm{~dB}$ for dropped signals. Finally, the second distribution stage, emulated by means of a variable optical attenuator (VOA), added $21 \mathrm{~dB}$ losses to the link, corresponding to $1: 128$ splitting.

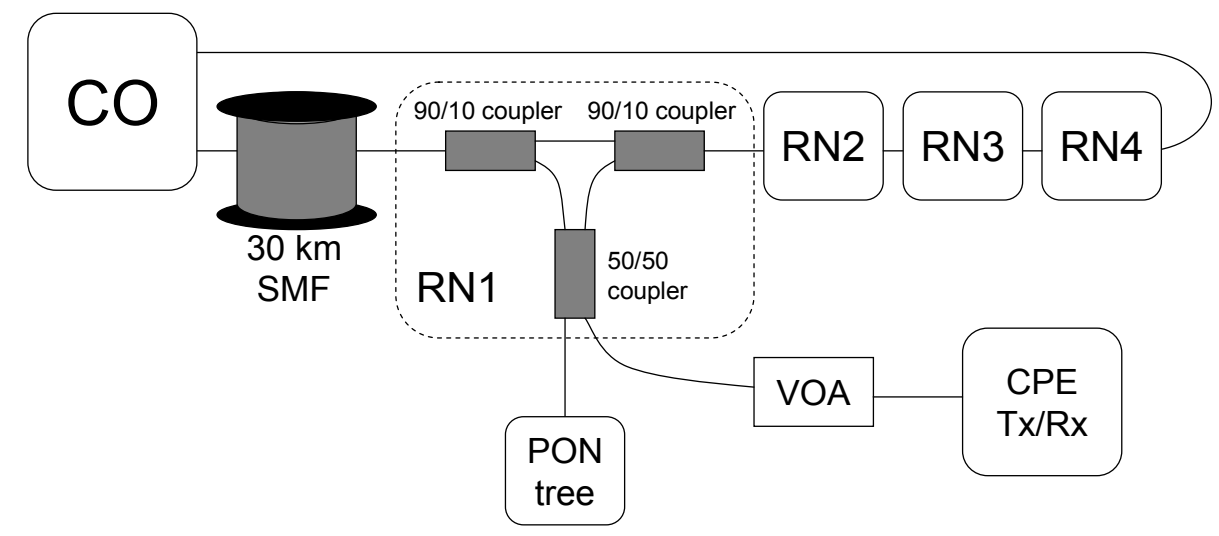

Figure 3. Experimental network testbed.

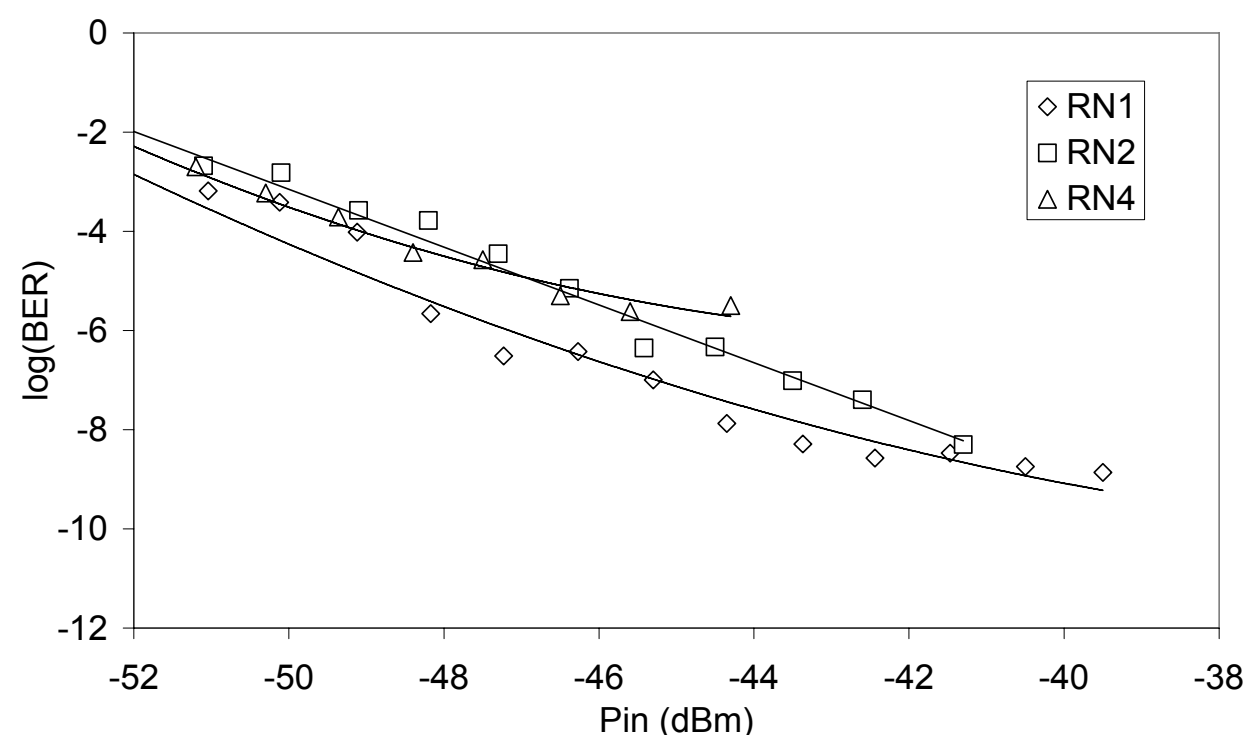

Figure 4. Transmission results.

The network tested had four remote nodes $(\mathrm{RN})$ thus total outside measured plant losses ranged from $39.4 \mathrm{~dB}$ on $\mathrm{RN} 1$ to $44.2 \mathrm{~dB}$ on RN4. In standard operation, the optical switch at the head-end would be configured to connect the users to the light path with less losses. The case of passing through three remote nodes to reach RN4 simulates the network working in resilient mode after a fiber break in the worst case (between the head-end and RN 4).

Three different cases were investigated: RN1, RN2 and RN4. RN2 represents the worst case in standard operation and RN4 the worst case in resilient mode. Results are shown in Fig. 4.

Regarding sensitivities, for a Bit Error Rate (BER) of $10^{-9}$ in RN1 sensitivity was measured to be $-43 \mathrm{dBm}$ and in RN2 a sensitivity of $-41.3 \mathrm{dBm}$ was obtained. Thus, the system performed correctly in standard operation mode.

In resilient mode, we could not reach a BER of $10^{-9}$ at RN4 due to a BER-floor. That floor is explained as deriving from the linewidth of the system $(1 \mathrm{MHz})$, and the frequency response distortion of the microwave mixers used at the reception side of the transceiver (see Fig. 2). Nevertheless, a BER of $10^{-6}$ was measured at $-44.3 \mathrm{dBm}$. To compare with the other cases, in RN 1 BER of $10^{-6}$ was measured at $-48.1 \mathrm{dBm}$, whereas in RN2 it was measured at $-46.3 \mathrm{dBm}$. A Forward Error Correction (FEC) coding scheme, with only $7 \%$ overhead, is able to recover (quasi) error-free data for a raw BER below $10^{-3}$ [8]. So, FEC strategies should be applied to 
increase BER over $10^{-9}$ in resilient mode. In that case we can work with an increased sensitivity of $-49.1 \mathrm{dBm}$. Penalty to adjacent channels at 4-GHz channel spacing was measured to be $0.1 \mathrm{~dB}$, as expected [6].

Table I shows a summary of the experiments carried out. There we can see that the power budget for the best case (RN1) is of $42.9 \mathrm{~dB}$, including the channel spacing penalty. For the worst case in normal operation, power budget is of $41.2 \mathrm{~dB}$. At a first glance, we could think on doubling the number of users supported in RN1 and RN4 in order to equilibrate such power budget, while increasing the total number of users up to 1536 . In that case, for maintaining the total output power at the $\mathrm{CO}$, we should reduce each wavelength power to $-0.8 \mathrm{dBm}$. Consequently each power budged would be reduced by $0.8 \mathrm{~dB}$, and in RN2 link losses would become higher than the power budget. So, even the power budget is not equilibrated at all for the different cases, the maximum number of users to serve is 1024 , due to safety power limits.

Table 1. Power budget.

\begin{tabular}{l|ccc||ccc}
\hline \hline & \multicolumn{3}{|c||}{ Normal Operation } & \multicolumn{3}{c}{ Resilient mode } \\
\hline \hline & RN1 & RN2 & RN4 & RN1 & RN2 & RN4 \\
\hline Sensitivity & $-43 \mathrm{dBm}$ & $-41.3 \mathrm{dBm}$ & - & $-49.1 \mathrm{dBm}$ & $-49.3 \mathrm{dBm}$ & $-49.1 \mathrm{dBm}$ \\
Link Losses & $39.4 \mathrm{~dB}$ & $41 \mathrm{~dB}$ & $44.2 \mathrm{~dB}$ & $39.4 \mathrm{~dB}$ & $41 \mathrm{~dB}$ & $44.2 \mathrm{~dB}$ \\
Power Budget & $42.9 \mathrm{~dB}$ & $41.2 \mathrm{~dB}$ & - & $49 \mathrm{~dB}$ & $49.2 \mathrm{~dB}$ & $49 \mathrm{~dB}$ \\
\hline \hline
\end{tabular}

\section{CONCLUSIONS}

This combined ring -tree access network topology has been demonstrated to provide a flexible and scalable architecture with large capabilities in terms of number of users (up to 1024) or in capacity (more than $1 \mathrm{~Tb} / \mathrm{s}$ ). Also, it features a completely passive outside plant, wavelength-transparent remote nodes and high transmission capabilities and resilience.

Transmission experiments at $1 \mathrm{~Gb} / \mathrm{s}$ show a sensitivity of $-43 \mathrm{dBm}$ in the first $\mathrm{RN}$, after $30 \mathrm{~km}$ and a power budget of $42.9 \mathrm{~dB}$. For the worst case (RN4), when the network is operating in resilient mode, we could reach BER better than $10^{-9}$ by applying FEC strategies. In that case a sensitivity of $-49.1 \mathrm{dBm}$ was achieved, showing a power budget of $49 \mathrm{~dB}$. Thus, we could guarantee $1 \mathrm{~Gb} / \mathrm{s}$ per user, achieving a total network capacity of $1024 \mathrm{~Gb} / \mathrm{s}$.

\section{ACKNOWLEDGEMENTS}

Authors want to thank Dr. Carlos Bock and Dr. Ronald Freund for their support and fruitful discussions.

\section{REFERENCES}

[1] J. A. Lázaro, C. Bock, V. Polo, R. I. Martinez and J. Prat, "Remotely amplified combined ring-tree dense access network architecture using reflective RSOA-based ONU," OSA Journal of Optical Networking, vol. 6, no. 6, pp. 801-807, Jun. 2007.

[2] C.-H. Lee, W. V. Sorin, and B. Y. Kim, "Fiber to the home using a PON infrastructure," IEEE Journal of Lightwave Technology, vol. 14, no. 12, pp. 4568-4683, Dec. 2006.

[3] L. G. Kazovsky, G. Kalogerakis, and W. T. Shaw, "Homodyne phase-shift-keying systems: Past challenges and future opportunities," IEEE Journal of Lightwave Technology, vol. 14, no. 12, pp. 4876-4884, Dec. 2006.

[4] J. M. Fàbrega, and J. Prat, "Homodyne receiver prototype with time-switching phase diversity and feedforward analog processing," OSA Optics Letters, vol. 32, no. 5, pp. 463-465, Mar. 2007.

[5] A. Ramaswamy, L. A. Johansson, J. Klamkin, C. Sheldon, H. F. Chou, M. J. Rodwel, L. A. Coldren, and J. E. Bowers "Coherent Receiver based on a Broadband Phase-Lock Loop," in 2007 Proc. OSA OFC, postdeadline paper.

[6] J. M. Fàbrega, and J. Prat, "Channel crosstalk in ultra-dense WDM PON using time-switched phase diversity optical homodyne reception," in Proc. ICTON 2007, paper Tu.A1.3.

[7] IEC Criteria on Safety for Optical Fiber Communication Systems, IEC Standard 60825-2, 2008.

[8] T. Mizuochi, Y. Miyata, T. Kobayashi, K. Ouchi, K. Kuno, K. Kubo, K. Shimizu, H. Tagami, H. Yoshida, H. Fujita, M. Akita, and K. Motoshima, "Forward error correction based on block turbo code with 3-bit soft decision for 10-Gb/s optical communication systems," IEEE Journal of Selected Topics in Quantum Electronics, vol. 10, no. 2, pp. 376-386, Feb. 2004. 